\title{
BMJ Open Different suturing techniques in thoracic incision: protocol for a feasibility randomised controlled trial
}

\author{
Zonghui Liu, ${ }^{1,2}$ Xiangyu Liu, ${ }^{1}$ Lin He, ${ }^{1}$ Xueyuan $\mathrm{Yu},{ }^{1}$ Lu Wang, ${ }^{1}$ Rui Wang, ${ }^{1}$ \\ Youcheng He, ${ }^{1}$ Xiaoyan Hao, ${ }^{1}$ Zhishui Tang, ${ }^{1}$ Yingjun Su, ${ }^{2}$ Maoguo Shu ${ }^{1}$
}

To cite: Liu Z, Liu X, He L, et al. Different suturing techniques in thoracic incision: protocol for a feasibility randomised controlled trial. BMJ Open 2019;9:e21645. doi:10.1136/ bmjopen-2018-021645

- Prepublication history for this paper is available online. To view these files, please visit the journal online (http://dx.do org/10.1136/bmjopen-2018021645).

YS and MS contributed equally.

Received 13 January 2018 Revised 31 October 2018 Accepted 8 November 2018

Check for updates

(c) Author(s) (or their employer(s)) 2019. Re-use permitted under CC BY-NC. No commercial re-use. See rights and permissions. Published by BMJ.

${ }^{1}$ Department of Aesthetic, Plastic and Maxillofacial Surgery, The First Affiliated Hospital of Xi'an Jiaotong University, Xi'an, Shaanxi, China

${ }^{2}$ Department of Plastic Surgery, Xijing Hospital, Fourth Military Medical University, Xi'an, Shaanxi, China

Correspondence to Dr Yingjun Su; xjzx2011@fmmu.edu.cn and

Dr Maoguo Shu

Shumaoguo@163.com

\section{ABSTRACT}

Introduction Based on the principles of the ideal skin closure technique, we previously described a suture technique (wedge-shaped excision and modified buried vertical mattress suture (WE-MBVMS)) that could provide excellent outcomes for the most demanding surfaces. However, adequate clinical comparative evidence supporting improved outcomes is lacking. Thus, the purpose of this protocol is to establish the feasibility of conducting a fully randomised controlled trial (RCT) comparing the clinical effectiveness of WE-MBVMS with a buried intradermal suture (BIS) in closing thoracic incision. Methods and analysis This study is a feasibility RCT of WE-MBVMS and BIS in patients undergoing surgery for costal cartilage harvesting. Seventy-eight participants are expected to participate in the study and will be randomised in a ratio of 1:1 to WE-MBVMS or BIS. Trial feasibility will be assessed by the number of participants assessed for eligibility, recruitment rates, reasons for ineligibility or non-participation, time for interventions, withdrawal and retention at all follow-up points (3, 6 and 12 months), follow-up rates and reasons for withdrawing from the trial. In addition, clinical data regarding the cosmetic results of scars will be collected to inform the sample size for a fully powered RCT.

Ethics and dissemination This study has been approved by The First Affiliated Hospital of Xi'an Jiaotong University Institutional Review Board (XJTU1AF2017LSK-120). The findings will be published in peer-reviewed journals. Trial registration number

\section{INTRODUCTION}

\section{Background}

Microtia is a congenital anomaly of the auricle, including partial or total defects of the ear. The prevalence ranges from 0.83 to 17.4 per 10000 births among regions, with a higher rate among Asians. ${ }^{1}$ Autogenous costal cartilage grafts have been an important method for microtia repair since they were described by Brent for ear reconstruction in $1920 .^{2}$ After the costal cartilage is harvested, the incision remains. Because the anterior chest is the most common keloid-bearing site, the scars resulting from these thoracic incisions may become keloid and therefore

\section{Strengths and limitations of this study}

This study is a feasibility randomised controlled trial to assess the feasibility and acceptability of wedge-shaped excision and modified buried vertical mattress suture (WE-MBVMS) for skin closure of a thoracic incision.

- By using feasibility outcome measures and clinical outcome measures, the results will provide more information regarding the clinical effectiveness of WE-MBVMS for a future large-scale RCT.

- An appropriate primary outcome measure will be selected to inform the sample size for a full RCT through examining the potential of scar assessment tools.

- A single centre will limit the generalisability of the results.

be aesthetically undesirable or symptomatic. ${ }^{3}$ The technique and material used for repair will influence the cosmetic appearance of the scar. $^{45}$

The ideal skin closure technique should ensure adequate haemostasis, reduce tension on the skin edges, approximate and evert the wound edges precisely, be relatively fast for surgeons to perform, be easy to care for and leave no suture marks. ${ }^{56}$ A method for dermal suturing, wedge-shaped excision and modified buried vertical mattress suture (WE-MBVMS), was described in 2009 based on summaries of clinical experience to achieve these goals. ${ }^{7}$ The following main characteristics distinguished this technique from other methods: (1) after the wedgeshaped excision was performed, the contact area of wound edges increased, enabling easy achievement of wound eversion and (2) the stitch was embedded in the dermis as long as possible to maximise the dermis area available for recruitment in the suture loop, forming visible skin folds and eversion. Thus, in theory, the method was successful at precisely approximating the edges of deep defects, relieving skin tension. ${ }^{7}$ Tension is considered 


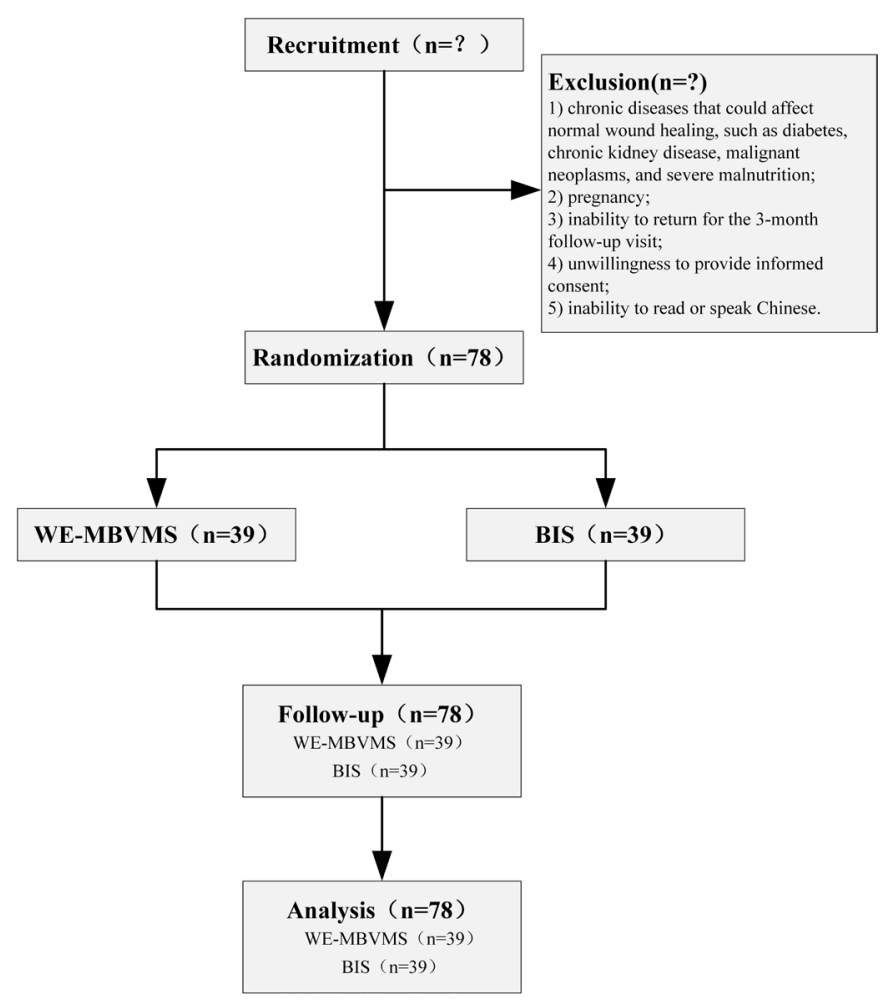

Figure 1 Flow chart depicting the trial protocol.

critical for the formation of hypertrophic scars ${ }^{3}$; therefore, WE-MBVMS might provide excellent outcomes for the most demanding surfaces. However, adequate comparative clinical evidence supporting improved outcomes with this technique has been limited. ${ }^{7}$

The traditional technique, buried intradermal suture (BIS), can provide prolonged dermal support without everting the wound edge and has been used for many years with good results. ${ }^{8}$ To date, WE-MBVMS and BIS have not been compared. Therefore, we propose to compare the cosmetic results of wounds/scars achieved with both closure techniques. However, published clinical evidence on WE-MBVMS is lacking; thus, a feasibility trial is required to produce more evidence for a fully powered randomised controlled trial (RCT).

\section{Objectives}

The primary objective is to investigate the feasibility of WE-MBVMS and BIS for patients undergoing costal cartilage harvesting in terms of patient recruitment, intervention and acceptability. The secondary objective is to observe the appearances achieved immediately postoperatively and cosmetic outcomes at all follow-up points (3, 6 and 12 months) achieved with WE-MBVMS and BIS.

\section{METHODS}

A flowchart of study recruitment, randomisation, follow-up and analysis is shown in figure 1 .

\section{Trial design}

This study is a single-centre feasibility RCT comparing the clinical effectiveness of WE-MBVMS and BIS to inform a future full-scale RCT.

\section{PARTICIPANTS \\ Inclusion criteria}

The inclusion criteria are (1) surgical incisions that must be closed after costal cartilage harvesting for reconstructive surgery (ears, noses, etc), (2) incisions at least $1 \mathrm{~cm}$ in length and (3) patients aged 18-60 years old.

\section{Exclusion criteria}

The exclusion criteria are (1) chronic diseases that could affect normal wound healing, such as diabetes, chronic kidney disease, malignant neoplasms and severe malnutrition; (2) pregnancy; (3) inability to return for the 3 month follow-up visit; (4) unwillingness to provide informed consent; and (5) inability to read or speak Chinese.

\section{Setting and location}

The trial will be implemented in the Department of Aesthetics, Plastic and Maxillofacial Surgery, The First Affiliated Hospital of Xi'an Jiaotong University.

\section{Surgeon expertise}

All surgeons (attending physician, fellow and residents) involved in the study have been trained by the senior author in suture techniques on pig skin to master both suture techniques. During the operation, the suture procedure will be further directed and approved by the author. The main researchers in the trial have successfully completed good clinical practice (GCP) training. All surgery practitioners have received qualifications as practising physicians and have at least 3 years of surgical experience. Before recruitment, the surgeons will attend a seminar about the study to ensure that they strictly adhere to the protocol.

\section{Recruitment}

All participants are enrolled after obtaining ethical approval and registration. Potential participants are screened for eligibility by clinicians. When a patient is recruited, general information, including his/her name, ID, sex, age, race, anamnesis, height and weight, is recorded in a secure, Web-based system. The research associate provides patients who meet the selection criteria with all of the study presentation materials. Patients have the opportunity to ask questions about the study and further discuss it with their families. Then, written informed consent is provided by patients before surgery.

\section{INTERVENTIONS}

After patients receive a general anaesthetic, experienced surgeons harvest the costal cartilage using a standardised technique. The soft periosteum, muscle and aponeurosis are closed by layering with 3-0 absorbable sutures (VICRYL Plus, Polyglactin 910; Johnson \& Johnson International, 

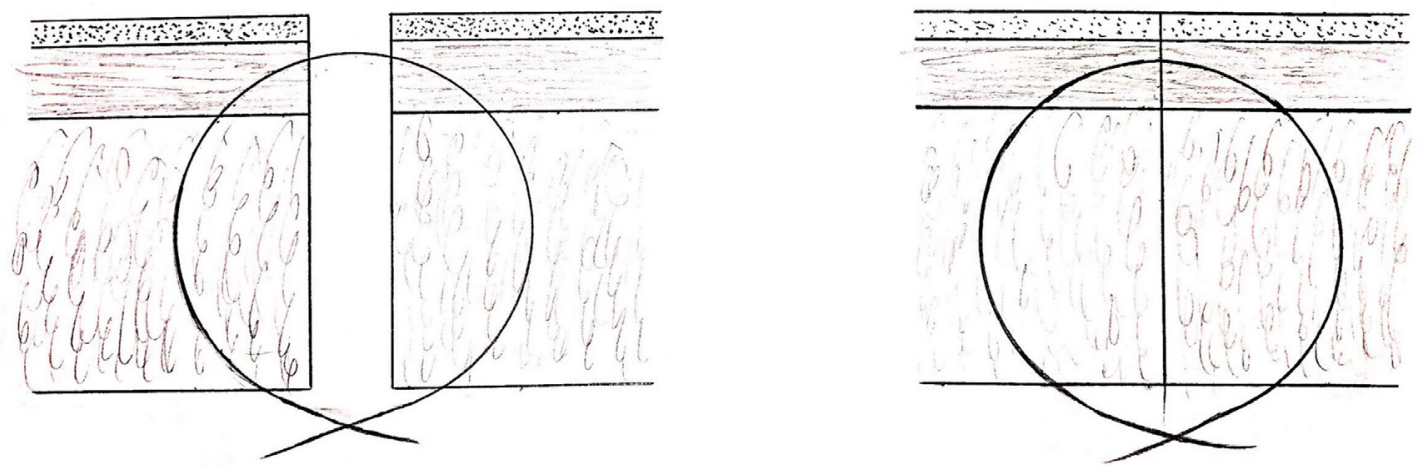

Figure 2 Cross-sectional view depicting the buried intradermal suture (BIS).

USA). Finally, the skin incision remains. Then, any skin contused during the operation is excised as cleanly as possible, and the wound edges are moderately undermined by the individual surgeon. Each incision is randomised to receive WE-MBVMS or BIS. An interrupted 4-0 absorbable suture (VICRYL Plus, Polyglactin 910; ETHICON, Johnson \& Johnson International, USA) is always placed for both suture techniques.

\section{Buried intradermal suture (BIS)}

The wound edges on both sides are perpendicular to the epidermis. The suture placement begins at the base of the flap, with one edge lifted by forceps. The stitch goes through the subcutaneous tissue and exits the dermis closest to the epidermis at the skin edge. At the other edge, the suture placement is performed in a mirrorimage fashion. Finally, the appearance of the wound is planar with no suture marks, and the knot buried. On the cross-section of the wound, the track of the suture forms a 'circle', as shown in figure $2 .{ }^{8}$

\section{Wedge-shaped excision and modified buried vertical mattress suture (WE-MBVMS)}

After more subcutaneous tissue is excised sharply, and the wedge-shaped excision is performed, both edges of the wound are tilted at a certain angle from the centre, forming an isosceles trapezoid on the cross-section of the wound. The suture placement also begins at the base of the flap.

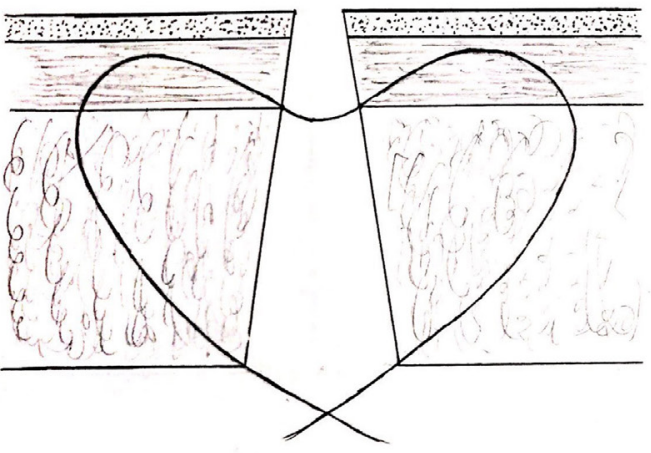

The stitch enters the dermis and moves along an arc track for as long as possible, and the superior arc is lower than the level of the midpapillary dermis. At the edge, the stitch exits from the subcutaneous-cutaneous boundary. At the other edge, suture placement is performed in a mirror-image fashion. Then, more dermis is recruited in a tight loop, with a knot tied at the base as with BIS. Finally, the appearance of the wound edge is everted, with folds surrounding it. On a cross-section of the wound, the track of the suture resembles a 'heart', as shown in figure $3{ }^{7}$

After BIS or WE-MBVMS has been performed, the wound is closed by intracutaneously running sutures with 5-0 monofilament absorbable sutures (MOMOCRYL, Poliglecaprone 25; ETHICON, USA) and adhesive strips (Steristrips, 3M Healthcare, USA) are placed at the end of surgery. Surgical dressing will be applied to the wound. Postoperative suggestions and medications will be identical for all wounds. A nurse will disinfect the wound with iodophor and replace the adhesive strips and surgical dressing once every 3 days for 9 days.

\section{OUTCOME MEASURES}

\section{Feasibility outcome measures}

The outcomes for the feasibility trial are descriptive ${ }^{9}$ and include the following: the number of participants assessed for eligibility and recruitment rates, reasons for

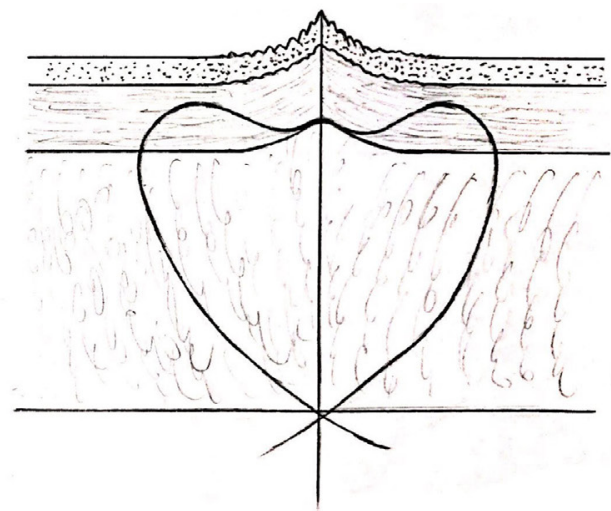

Figure 3 Cross-sectional view depicting the wedge-shaped excision and modified buried vertical mattress suture (WEMBVMS). 
ineligibility or non-participation, time for interventions, number of withdrawals and retention at all follow-up points ( 3 months, 6 months and 12 months) and follow-up rates, and reasons for withdrawing from the trial.

\section{Clinical outcome measures}

At baseline and all follow-up points, participants and researchers will be asked to complete questionnaires for data collection. In the feasibility trial, we will select the appropriate primary outcome measure for a future fullscale RCT from the following scar assessment tools: the Vancouver Scar Scale (VSS), Patient and Observer Scar Assessment Scale (POSAS) and Visual Analogue Scale (VAS), as well as the width of the scars at all follow-up points.

In a review of scar rating scales by Zephanie et al, the POSAS exhibited superior performance with high-quality reliability, while the VSS has undergone the most thorough review among clinimetrics. ${ }^{10}$ Jonathan et al showed that the VAS is consistent, reliable, valid and feasible for assessing overall satisfaction with scar cosmesis. ${ }^{11}$ The VSS has four separate domains, vascularity, pigmentation, pliability and height. Each item has ranked subscales, and the total score, obtained by summation, ranges from 0 to 13 , with 0 representing a normal appearance and 13 representing the worst. ${ }^{12}$ The POSAS consists of two scales, the Patient Scar Assessment Scale (PSAS) and Observer Scar Assessment Scale (OSAS). The PSAS has the following six domains: pain, itching, colour, pliability, thickness and relief. The OSAS also contains six items, including vascularity, pigmentation, thickness, relief, pliability and surface area. ${ }^{13} 14$ Each of the six items is graded on a 10-point scale, with 1 indicating normal skin and 10 indicating the worst disfiguring scar. The summary score ranges from 6 to 60 , with 60 indicating the worst imaginable scar. ${ }^{13} 14$ The VAS is a $10 \mathrm{~cm}$ line, with 0 indicating the worst and 10 indicating the best overall satisfaction. ${ }^{15}$ Patients are asked to place a vertical mark on the VAS to rate the appearance of their scars.

The other outcomes are the appearance of the wound achieved immediately postoperatively and the complication rates during the 12-month follow-up period. The postoperative appearance of the wound includes the eversion height and width of the wound edges as measured by a ruler. Wound-related complications include surgical incision dehiscence, seroma or haematoma, infection and spitting sutures, which are recorded in detail.

\section{Qualitative outcomes}

The acceptability of the interventions will be examined through a qualitative approach. We will conduct qualitative interviews with the participants by using a topic guide to ascertain perceptions about acceptability. During the qualitative interviews, we will gather a range of information on topics including responses to wounds/scars, the acceptability of the randomisation procedure and methods of data collection and adherence to treatment and follow-up.
Table 1 Follow-up measures

\begin{tabular}{|c|c|}
\hline Time point & Data collected \\
\hline Baseline & $\begin{array}{l}\text { Name, ID, sex, age, race, } \\
\text { smoking history, height and } \\
\text { weight, number of participants } \\
\text { assessed for eligibility and } \\
\text { recruitment rates, reasons for } \\
\text { ineligibility or non-participation. }\end{array}$ \\
\hline $\begin{array}{l}\text { Immediately } \\
\text { postoperatively }\end{array}$ & $\begin{array}{l}\text { Eversion height and width of } \\
\text { wound edges and time for } \\
\text { interventions. }\end{array}$ \\
\hline $\begin{array}{l}\text { During the } 12 \text {-month } \\
\text { follow-up period }\end{array}$ & $\begin{array}{l}\text { Number of complications (such } \\
\text { as surgical incision dehiscence, } \\
\text { seroma or haematoma, infection } \\
\text { and spitting of sutures). }\end{array}$ \\
\hline $\begin{array}{l}3 \text { month, } 6 \text { month and } \\
12 \text { month follow-up }\end{array}$ & $\begin{array}{l}\text { Scores on the VSS, VAS and } \\
\text { POSAS; width of scars; number } \\
\text { of withdrawals and retention } \\
\text { and follow-up rates; reasons for } \\
\text { withdrawing from the trial. }\end{array}$ \\
\hline
\end{tabular}

POSAS, Patient and Observer Scar Assessment Scale; VAS, Visual Analogue Scale; VSS, Vancouver Scar Scale.

\section{Follow-up}

Over time, the number of drop-outs will increase. In a study by James et al, the wound scores of scars at 1 year were strongly associated with those at 3 months. ${ }^{16}$ In similar studies about scars, ${ }^{17-20}$ a difference in cosmetic outcomes was already detected 3 months after interventions. At 3 months after interventions, a subcutaneous absorbable suture (VICRYL Plus, Polyglactin 910) is almost completely absorbed ${ }^{5}$; then, the effect of reduced tension will gradually disappear, and differences in surgical interventions will tend to diminish. Therefore, we will set the first follow-up point at 3 months. For observing changes in the appearance of scars, we will continue the follow-up to 6 months and 12 months when the scars have matured. ${ }^{21}$

During the follow-up period, patients will receive comprehensive care. If complications occur, medical treatment will be administered. Wound data collected immediately postoperatively and complications during follow-up will be measured or recorded by dedicated personnel. At all follow-up points, patients and two blinded observers will be asked to complete the scales for the cosmetic outcomes of scars administered by a professional questionnaire administrator. The appearance of incisions immediately postoperatively and at all follow-up points will be recorded by digital photographs. The schedule of follow-up measures is presented in table 1 .

\section{Data management}

Data, including baseline information about the patients, intervention procedures and outcomes, will be recorded via a standard data collection and management system, including case report forms (CRFs) and electronic data capture (ResMan online, http://www.medresman. org/uc/index.aspx). The patient's name on the CRF is 
identified by initials to maintain his or her anonymity. All of the records are identified only by the patient's ID and initials. The data are logged by two independent researchers, and the data manager further checks the integrity of the data to reduce the error rate.

\section{Sample size}

As this is a feasibility study, formal power calculations were not carried out. ${ }^{22}$ Thus, we calculated the sample size mainly with reference to similar clinical trials. ${ }^{172}{ }^{23}$ To detect a difference of $5(\beta=20 \%)$ on the 60 -point POSAS with a significance level $(\alpha)$ of 0.05 and a SD of 7 , we calculate that 32 patients per group are needed by GPower software V.3.1. ${ }^{23-25}$ Assuming a predicted dropout rate of 20\%, 39 patients per group are needed. Therefore, we plan to recruit 78 subjects.

\section{Randomisation}

A professional statistician will generate a random number sequence with SAS (V.9.4) statistical software in a 1:1 ratio before recruitment. The allocation assignment for participant randomisation is made in the random list and recruitment order.

\section{Blinding}

The randomisation list is placed in an opaque envelope and kept by a nurse who is not directly participating in the study. After a patient is recruited, the nurse consults the list and informs the surgeon of the treatment allocation only. Then, the envelope is sealed again by the nurse, and the list is remains concealed until the last patient has been recruited. The patients and observers who will assess the cosmetic outcomes of scars are blinded to the allocation. The patient knows only that one of two techniques will be applied randomly and does not know which specific one will be used. The OSAS will be completed by two blinded observers for validity.

\section{Statistical analyses}

In line with the recommendations of GCP in the analysis of feasibility studies, the analysis will be descriptive. ${ }^{26}$ Descriptive statistics will be calculated for feasibility outcomes including recruitment rates, follow-up rates, time for interventions and baseline characteristics. Descriptive statistics will also be calculated for clinical outcomes including eversion height and the width of wound edges immediately postoperatively, complication rate during the 12-month follow-up, POSAS, VSS, VAS and the width of scars at all follow-up points. Means and SDs or $95 \%$ CIs, medians and quartile ranges or percentages will be presented. We will use the SDs of outcome measures to develop estimates for the sample size in a fully powered RCT. As this study is a feasibility trial, the objective is not hypothesis testing; rather, these analyses allow for preliminary examination of trends in comparisons. ${ }^{27}$ The analysis will be undertaken on an intentionto-treat basis.

\section{Monitoring}

Because the follow-up is short, and the risks of intervention are clear, we have not established a data management committee. Adverse events will be monitored and recorded using the routine report system. Written informed consent documents, recruitment status and overall trial progress will be monitored by the Research Ethics Committee of The First Affiliated Hospital of Xi'an Jiaotong University.

\section{Patient and public involvement}

Patient representatives comprising patients and their families have been consulted for advice on the research proposal before implementation of the trial. By conducting qualitative interviews with representatives, we have developed the original research proposal and acquired a range of interviewing skills, including conducting interviews in places where children already feel comfortable, giving participants enough time to tell their stories and encouraging them to express themselves through drawings or play. They have agreed to become members of the steering committee and will be involved in the recruitment and conduct of the study. Feedback and comments from representatives at all stages of the project will contribute substantially to exploring the findings of the study. The results of the trial will be summarised and disseminated to the participants by social media, a network platform, posters, diagrams and conference presentations.

\section{Ethics and dissemination}

The study will be conducted in conformity with the Declaration of Helsinki and the Chinese laws and regulations regarding clinical trials. All the participants will provide written informed consent to participate in the study before recruitment. At any time during this trial, participants will have the power to withdraw without any impact on the quality of usual care. Any amendments to the protocol will be communicated to the participants, practitioners, institutional review board and registration authority if deemed necessary. The findings of this study will be published in peer-reviewed journals and presented at national and international scientific conferences. Reports will follow international guidelines, including the Consolidating Standards of Reporting Trials Statement and Standard Protocol Items: Recommendations for Interventional Trials 2013 Statement. The authors will be individuals who have made meaningful contributions to the research.

\section{DISCUSSION}

Good cosmetic appearance of scars has always been desired by surgeons and patients. For this purpose, we previously described WE-MBVMS by summarising our clinical experience. ${ }^{7}$ Although two patients with hypertrophic scars were cured by this technique, data regarding comparative clinical outcomes are lacking. This study will be conducted to investigate the feasibility of a full RCT to assess the clinical effectiveness of WE-MBVMS and BIS in the chest. This feasibility RCT will help us to select an 
appropriate primary clinical outcome measure from the scar assessment tools to inform the sample size for a full RCT.

All detailed descriptions of the appearances of wounds / scars will help us to gain a better understanding of both suture techniques. In a study by Moody et al, scars achieved with running horizontal mattress sutures appeared smoother and flatter than those achieved with simple running sutures. ${ }^{28}$ Therefore, the authors concluded that excellent wound edge eversion was significantly associated with improved cosmetic outcomes of scars. ${ }^{28}$ However, Kappel et al found no such association when comparing everted closures to planar closures. ${ }^{29}$ Wang et al showed that set-back sutures provided significantly better wound eversion than did buried vertical mattress sutures and produced superior cosmetic outcomes. ${ }^{17}$ According to Trufant and Leach, site selection and technical levels may be confounding factors in the differences reported by Kappel et al and Wang et al. ${ }^{30}$ Hence, whether wound edge eversion is beneficial remains controversial. If feasibility is demonstrated, our future research evaluating both techniques on a single surgical site may provide more evidence to settle this dispute as WE-MBVMS is a dermal suturing technique that can produce wound edge eversion. This study is limited by its single-centre context. However, we believe that the findings will be very valuable for the design of a future full-scale trial.

Acknowledgements The authors acknowledge Dian Zhu (data manager) for support before the trial. The authors are also grateful to the participants and the members of the patient and public involvement group for their invaluable advice.

Contributors MS, ZL and YS conceived and designed the trial. MS and ZL are the principal researchers and wrote the first draft. MS is the responsible sponsor. LW, RW and YH helped to revise the "Inclusion and exclusion criteria" section. XH and ZT helped to modify the "Interventions" section. XL, LH and XY contributed to the revision of the "Outcome" section. LW, ZL and YH will recruit patients in the hospital and conduct qualitative interviews. XH and ZT will perform surgical operations. LH and $X Y$ will assess the cosmetic outcomes of the scars. $X L$ and RW will collect and record data. All designated authors have contributed to the design and revision of the protocol, and all the authors have carefully read and approved the final manuscript.

Funding The authors have not declared a specific grant for this research from any funding agency in the public, commercial or not-for-profit sectors.

Competing interests None declared.

Patient consent for publication Obtained.

Ethics approval The First Affiliated Hospital of Xi'an Jiaotong University Institutional Review Board (XJTU1AF2017LSK-120).

Provenance and peer review Not commissioned; externally peer reviewed.

Open access This is an open access article distributed in accordance with the Creative Commons Attribution Non Commercial (CC BY-NC 4.0) license, which permits others to distribute, remix, adapt, build upon this work non-commercially, and license their derivative works on different terms, provided the original work is properly cited, appropriate credit is given, any changes made indicated, and the use is non-commercial. See: http://creativecommons.org/licenses/by-nc/4.0/.

\section{REFERENCES}

1. Luquetti DV, Heike CL, Hing AV, et al. Microtia: epidemiology and genetics. Am J Med Genet A 2012;158A:124-39.

2. Brent $B$. Auricular repair with autogenous rib cartilage grafts: two decades of experience with 600 cases. Plast Reconstr Surg 1992;90-355-74.
3. Ogawa R, Okai K, Tokumura F, et al. The relationship between skin stretching/contraction and pathologic scarring: the important role of mechanical forces in keloid generation. Wound Repair Regen 2012;20:149-57.

4. Odijk R, Hennipman B, Rousian M, et al. The MOVE-trial: Monocryl ${ }^{\circledR}$ vs. Vicryl Rapide ${ }^{\mathrm{TM}}$ for skin repair in mediolateral episiotomies: a randomized controlled trial. BMC Pregnancy Childbirth 2017;17:355.

5. Regula CG, Yag-Howard C. Suture products and techniques. Dermatologic Surgery 2015;41:S187-S200.

6. Yag-Howard C. Sutures, Needles, and Tissue Adhesives. Dermatologic Surgery 2014;40:S3-S15.

7. Zhang X, Diao JS, Guo SZ, et al. Wedge-shaped excision and modified vertical mattress suture fully buried in a multilayered and tensioned wound closure. Aesthetic Plast Surg 2009;33:457-60.

8. Zitelli JA, Moy RL. Buried vertical mattress suture. J Dermatol Surg Oncol 1989;15:17-20.

9. Orsmond Gl, Cohn ES. The Distinctive Features of a Feasibility Study. OTJR: Occupation, Participation and Health, 2015;35: 169-77.

10. Tyack Z, Simons M, Spinks A, et al. A systematic review of the quality of burn scar rating scales for clinical and research use. Burns 2012;38:6-18.

11. Duncan JAL, Bond JS, Mason T, et al. Visual Analogue Scale Scoring and Ranking: A Suitable and Sensitive Method for Assessing Scar Quality? Plast Reconstr Surg 2006;118:909-18.

12. Baryza MJ, Baryza GA. The Vancouver Scar Scale: an administration tool and its interrater reliability. J Burn Care Rehabil 1995;16:535-8.

13. van de Kar AL, Corion LU, Smeulders MJ, et al. Reliable and feasible evaluation of linear scars by the Patient and Observer Scar Assessment Scale. Plast Reconstr Surg 2005;116:514-22.

14. Draaijers LJ, Tempelman FR, Botman YA, et al. The patient and observer scar assessment scale: a reliable and feasible tool for scar evaluation. Plast Reconstr Surg 2004;113:1960-5.

15. Duncan JA, Bond JS, Mason T, et al. Visual analogue scale scoring and ranking: a suitable and sensitive method for assessing scar quality? Plast Reconstr Surg 2006;118:909-18.

16. Quinn J, Wells G, Sutcliffe T, et al. Tissue adhesive versus suture wound repair at 1 year: randomized clinical trial correlating early, 3-month, and 1-year cosmetic outcome. Ann Emerg Med 1998;32:645-9.

17. Wang AS, Kleinerman R, Armstrong AW, et al. Set-back versus buried vertical mattress suturing: results of a randomized blinded trial. J Am Acad Dermatol 2015;72:674-80.

18. Park YJ, Kim SJ, Song HS, et al. Prevention of thyroidectomy scars in asian adults with low-level light therapy. Dermatologic Surgery 2016;42:526-34.

19. Simforoosh N, Abedi A, Hosseini Sharifi SH, et al. Comparison of surgical outcomes and cosmetic results between standard and mini laparoscopic pyeloplasty in children younger than 1 year of age. $J$ Pediatr Urol 2014;10:819-23.

20. Luck R, Tredway T, Gerard J, et al. Comparison of cosmetic outcomes of absorbable versus nonabsorbable sutures in pediatric facial lacerations. Pediatr Emerg Care 2013;29:691-5.

21. Reish RG, Eriksson E. Scar treatments: preclinical and clinical studies. J Am Coll Surg 2008;206:719-30.

22. Arain $\mathrm{M}$, Campbell MJ, Cooper CL, et al. What is a pilot or feasibility study? A review of current practice and editorial policy. BMC Med Res Methodol 2010;10:67.

23. Cromi A, Ghezzi F, Gottardi A, et al. Cosmetic outcomes of various skin closure methods following cesarean delivery: a randomized trial. Am J Obstet Gynecol 2010;203-36.e1-36.e8.

24. Custis T, Armstrong AW, King TH, et al. Effect of adhesive strips and dermal sutures vs dermal sutures only on wound closure. JAMA Dermatol 2015;151:862.

25. Faul F, Erdfelder E, Lang AG, et al. G*Power 3: a flexible statistical power analysis program for the social, behavioral, and biomedical sciences. Behav Res Methods 2007;39:175-91.

26. Lancaster GA, Dodd S, Williamson PR. Design and analysis of pilot studies: recommendations for good practice. J Eval Clin Pract 2004;10:307-12.

27. Whitehead AL, Sully BG, Campbell MJ. Pilot and feasibility studies: is there a difference from each other and from a randomised controlled trial? Contemp Clin Trials 2014;38:130-3.

28. Moody BR, McCarthy JE, Linder J, et al. Enhanced cosmetic outcome with running horizontal mattress sutures. Dermatol Surg 2005;31:1313-6.

29. Kappel S, Kleinerman R, King TH, et al. Does wound eversion improve cosmetic outcome? J Am Acad Dermatol 2015;72:668-73.

30. Trufant JW, Leach BC. Commentary: Wound edge eversion. J Am Acad Dermatol 2015;72:681-2. 